\title{
Open-Hardware and Application Specific Design for the Monitoring System of the Belle II Forward/Backward Electromagnetic Calorimeter
}

\author{
Francesco Di Capua ${ }^{1,2 *}$, Alberto Aloisio ${ }^{1,2}$, Fabrizio Ameli ${ }^{3}$, Antonio Anastasio ${ }^{2}$, Paolo \\ Branchini $^{4}$, Raffaele Giordano ${ }^{1,2}$, Vincenzo Izzo $^{2}$, Gennaro Tortone ${ }^{2}$ \\ ${ }^{1}$ University of Naples, "Federico II", Via Cinthia 80126 Napoli, Italy \\ ${ }^{2}$ Istituto Nazionale di Fisica Nucleare, Sez. di Napoli, Via Cinthia 80126 Napoli, Italy \\ ${ }^{3}$ Istituto Nazionale di Fisica Nucleare, Sez. di Roma, Piazz.le A. Moro 00100, Roma Italy \\ ${ }^{4}$ Istituto Nazionale di Fisica Nucleare, Sez. di Roma Tre, Via della Vasca Navale 00100, Roma Italy
}

\begin{abstract}
Control and monitoring of experimental facilities as well as laboratory equipment requires handling a blend of different tasks. Often in industrial or scientific fields there are standards or form factor to comply with and electronic interfaces or custom busses to adopt. With such tight boundary conditions, the integration of an off-the-shelf Single Board Computer (SBC) is not always a possible or viable alternative. The availability of electronic schematics and PCBs with open-source Hardware license for various SBCs overcomes such integration problems, making feasible the implementation of a custom architecture composed by a central core inherited from a vendor reference design (most likely the microprocessor, static RAM and flash memory) augmented with application-specific integrated circuits and hardware resources, in order to handle the requirements of the specific environment. The user is then able to exploit most of the supported tools and software provided by opensource community, fulfilling all the constraints enforced by his environment. We have used such an approach for the design and development of the monitoring system of the endcap electromagnetic calorimeter of the Belle II experiment, presently running at KEK Laboratory (Tsukuba, Japan). Here we present and discuss the main aspects of the hardware architectures and noise performances tailored on the needs of a detector designed around CsI crystal scintillators.
\end{abstract}

\section{Introduction}

In last years a wide selection of ARM controllers offered by several vendors feature high performances, low power consuming and very low cost. In addition such controllers can be easily interfaced with a huge set number of peripherals like analog coverters, serial-bus and network controllers, just to mention some of them. This hardware is directly supported by vendors in term of evaluation boards, code tools for analysis and tuning of hardware

* Corresponding author: dicapua@na.infn.it 
parameters. All these reasons makes ARM cores an ideal choice for the design of a new generation of embedded platforms for control and monitor of high-energy experiments in complex environments. In this paper we present the uSOP system, a Single Board Computer, based on a versatile and expandable miroprocessor, used in Belle II [1] experiment at KEK Laboratory for monitoring the endcap electromagnetic calorimeter. The uSOP board is based on the use of a powerful arm processor interfaced with a high performance data acquisition System-On-Chip. The uSOP PCB has been realized by using basic rules for Electromagnetic interference (EMI) immunity and low noise performances. All peripherals are galvanically isolated and provived with separate power supply. The 24bit LTC2983 high-resolution ADC, proving to guarantee noise floor in the range of $1 \mu \mathrm{V}$, has been implemented in the uSOP system. The noise rejection power capability within the uSOP system has been tested in laboratory, results and performances are described in the following.

\section{The Service Oriented Platform uSOP}

The system is based on the high performance ARM processor Sitara AM335x [2], a CortexA8 SoC produced by Texas Instruments. The uSOP design has been inherited by the BeagleBone [3] project from the BeagleBoard org Foundation [4], a non-profit corporation that promotes open-source hardware and software. The configuration adopted is a Sitara AM3358 microprocessor with up to 512 Mbyte RAM and 4 Gbyte Flash.

The uSOP board (Figure 1) communicates through the most used serial busses, like SPI, I2C and JTAG, which have been galvanically isolated. A 10/100 Ethernet port is available for networking and a Host and device USB ports are present. uSOP system has an independent network module Lantronix Xport Pro [5], a self-contained embedded Linux module that allows the user to perform system level critical operations (flashing or booting the operating system). A fully description of uSOP board hardware can be found in [6].

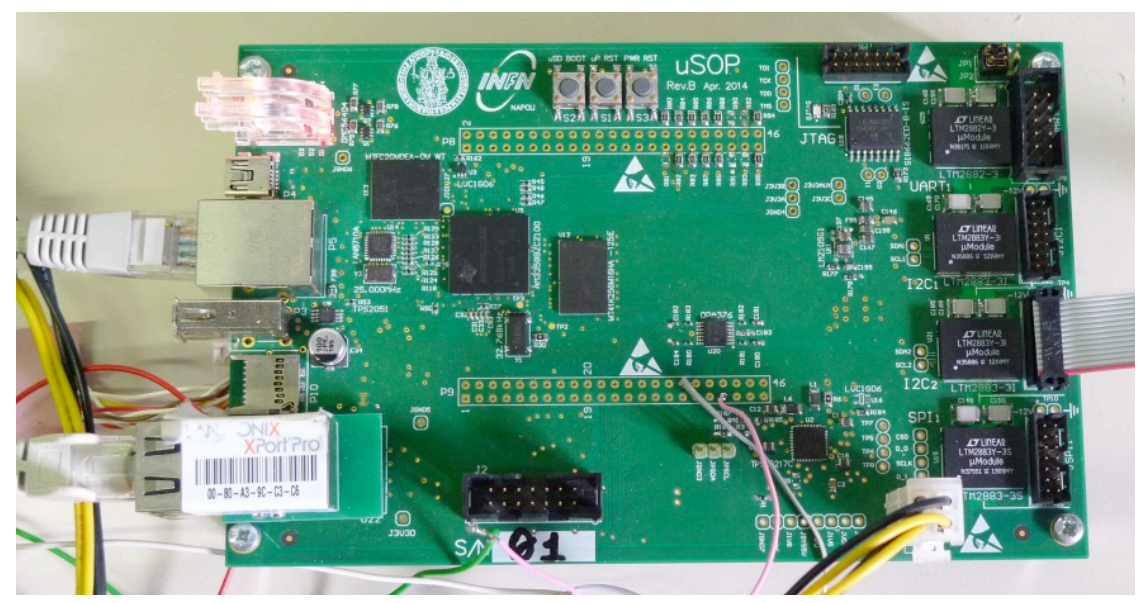

Figure 1. The uSOP board. 


\section{Application specific: the Belle II Electromagnetic Calorimeter}

The Belle II experiment is the successor to the Belle experiment and started taking data in early 2018. Belle II is expected to collect increased data than its predecessor due to 40 time increased instantaneous luminosity provided by SuperKEKB [7] electron-positron collider. The increased statistic will allow investigating the $\mathrm{CP}$-violating asymmetries in rare $\mathrm{B}$ mesons decays, the properties of CKM matrix and performing searches of new physics beyond the Standard Model, including studies in the Dark Sector [8].

\subsection{The Belle II Electromagnetic Calorimeter}

The Electromagnetic Calorimeter (ECL), consists in a barrel $3 \mathrm{~m}$ long with an inner radius of $1.25 \mathrm{~m}$ and two annular endcaps, named forward and backward. The ECL is based on CsI(Tl) scintillating crystals, which main characteristics are high light yield and short radiation length. The barrel contains 8736 crystals and the two endcaps contains 2112 crystals, distributed in 32 sectors [9].

Main drawback of $\mathrm{CsI}(\mathrm{Tl})$ crystals is the light yield variations as a function of temperature [10]. In addition, they can be permanently damaged by exposure at high humidity levels [11]. Due to such critical behaviour, a monitoring of environmental parameters for $\mathrm{CsI}(\mathrm{Tl})$ crystals during the full period of the experiment is essential.

\subsection{The T-Rh Controller}

In order to monitor environmental parameters, a specific controller has been designed. The developed board (Figure 2) is based on the LTC2983, a SoC with a 24-bit ADC, analog multiplexing current source and microprocessor-assisted linearization [12]. The device allows a 3-wire measurement scheme and the implementation of a measurement cycle aiming at the best dynamic range and at cancelation of parasitic thermocouple effects.

Each of the 32 sectors of ECL has been equipped with three Semitec AT-2 thermistors [13] and an active Vaisala HMP60 humidity probe [14]. In total 128 analog channels need to be read-out ( 96 thermistors +32 humidity probes). A T-Rh controller can read two ECL sectors. Two of them have been connected to uSOP board through SPI. As a consequence, 8 uSOP boards are required to acquire the complete detector. 


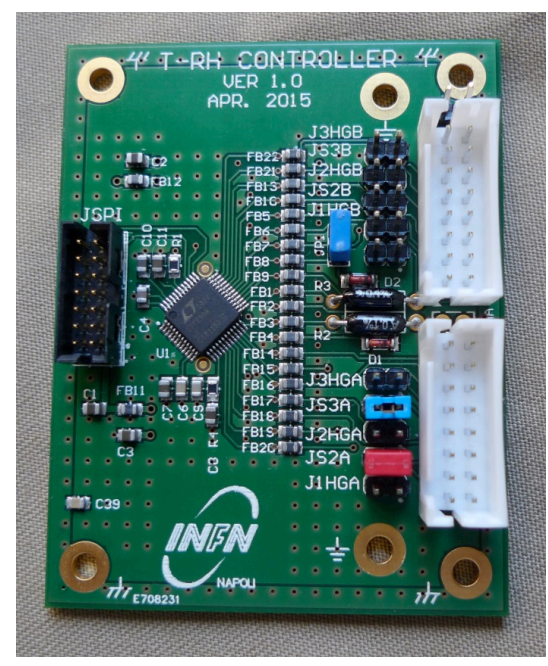

Figure 2. The T-Rh controller based on LTC2983 System-On-Chip.

\section{Noise Performances}

In order to validate the architecture in the Belle II environment, laboratory testing of the noise performance and rejection capability of the system have been carried out. We performed a measurement of the Common-Mode Rejection Ratio (CMRR), which represents a good figure of merit of the system performances in a noise environment.

In order to emulate the real application, the measurements have been performed with a 40 $m$ length cables to connect the common mode input voltage to the ADC. A CMRR of -135 $\mathrm{dB}$ has been obtained preserving the original ADC performances with a standard deviation of the noise floor of $0.7 \mu \mathrm{V}$ (Figure 3 ).

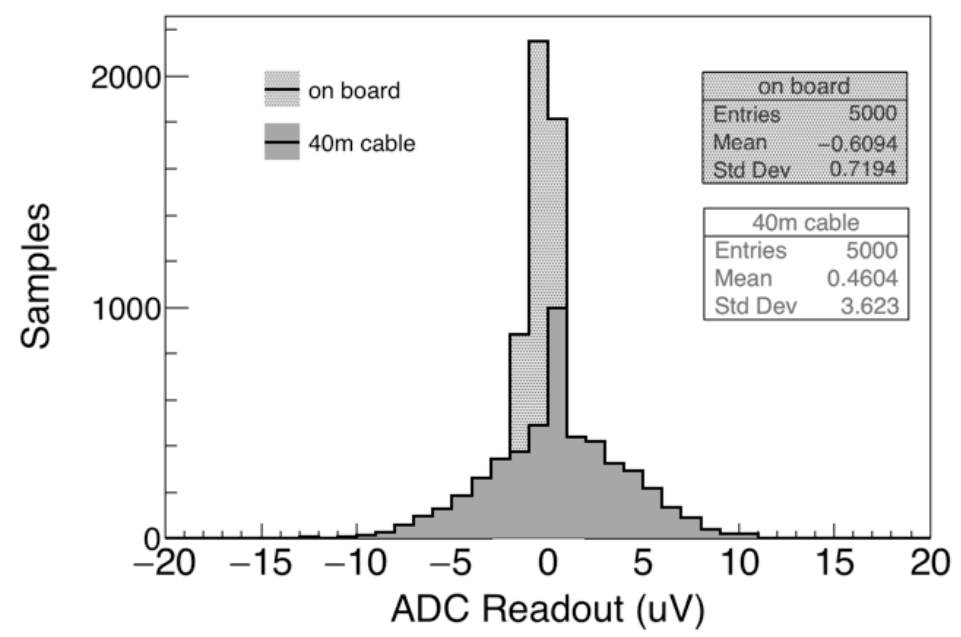

Figure 3. Common Mode at $1.1 \mathrm{~V}$ input with and without $40 \mathrm{~m}$ length cable. 
Low frequency 50-60 Hz noise can deteriorate the ADC performance. At this purpose, Normal Mode Rejection Ratio (NMRR) has been measured comparing the three different filters of the LTC2983: $50 \mathrm{~Hz}, 60 \mathrm{~Hz}, 50 \& \& 60 \mathrm{~Hz}$.

For such measurement, we used a function generator in order to keep the input voltage at center of the common mode input range. This has been implemented by friving two sinusoidal waveforms opposite in phase. The measurement scheme for NMRR is represented in Figure 4.
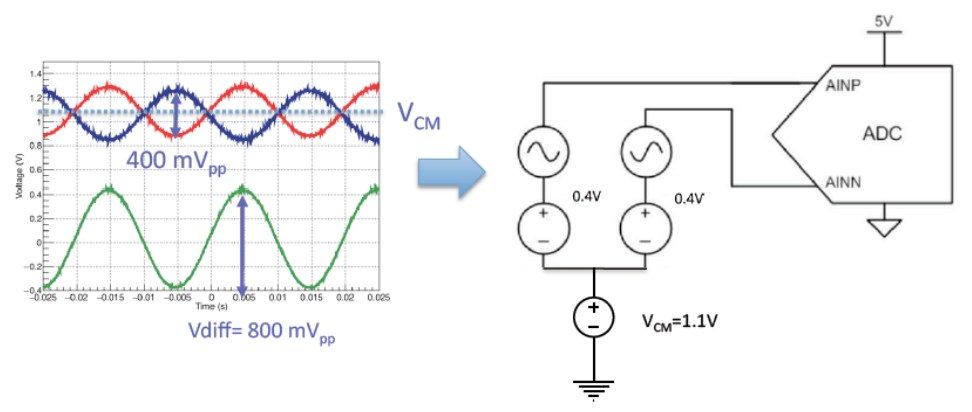

Figure 4. Normal Mode Rejection Ratio measurement scheme.

By changing the input voltage frequency, we verified that filters start to become effective from $40 \mathrm{~Hz}$ (Figure 5). The system shows an excellent noise rejection: an attenuation of 80 $\mathrm{dB}$ has been achieved (Figure 6).

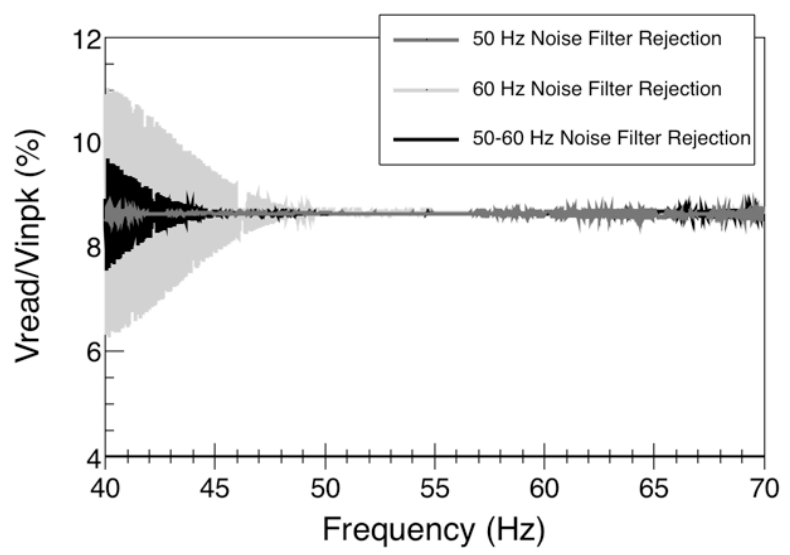

Figure 5. Residual fraction of the input signal as a function of the signal frequency. 


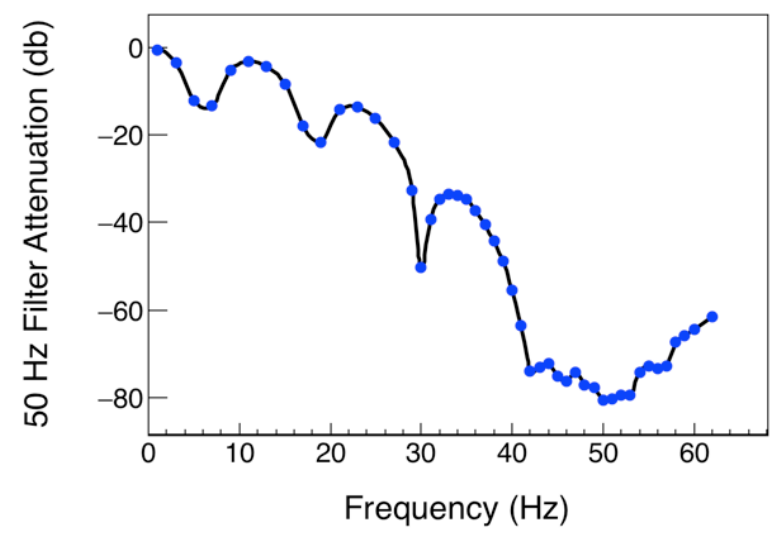

Figure 6. Normal Mode Rejection Ratio as a function of the input signal frequency.

\section{Conclusions}

uSOP is an Open-Hardware platform actually in use in Belle II experiment for monitoring of the environmental temperature and humidity of CsI crystals of the electromagnetic calorimeter. uSOP system can be fully managed remotely and shows excellent noise performances. The approach used and the service-oriented platform developed has shown to be a flexible and reliable solution for high-energy physics experiment

\section{References}

1. T. Abe et al., arXiv:1011.0352, KEK-REPORT 2010-1 (2010).

2. Sitara ${ }^{\mathrm{TM}}$ AM $335 \mathrm{x}$ Processors, Texas Instruments, http://www.ti.com/lsds/ti/processors/sitara/arm cortexa8/am335x/overview.page.

3. BeagleBoard Black, https://beagleboard.org/black.

4. About BeagleBoard.org and the BeagleBoard.org Foundation, http://beagleboard.org/about.

5. XPort Pro Embedded Device Server User Guide, Lantronix, https://www.lantronix.com/wp-content/uploads/pdf/900560e_XPort_Pro_UG_release.pdf.

6. A. Aloisio et al., IEEE TNS, 99, 1 1185-1190, (2017).

7. Y. Ohnishi et al., PTEP 2013, 03A011 (2013).

8. The Belle II Physics Book, KEK Preprint 2018-27, BELLE2-PUB-PH-2018-001, FERMILAB-PUB-18-398-T, JLAB-THY-18-2780, INT-PUB-18-047, UWThPh 201826, arXiv: 1808.10567 (2018).

9. V.E. Shebalin, Physics of Particle and Nuclei, 49, 4, 793-798 (2018).

10. ZHU Ren-Yuan., Nucl. Phys. B, 78, 203-219 (1999).

11. CHEN Ruo-Fu. et al., Chinese Phys. C, 32, 2 135-138 (2008).

12. LTC2983 - Multi-Sensor High Accuracy Digital Temperature Measurement System, http://www.linear.com/product/LTC2983. 
13. Semitec Corp.,"High Precision Thermistor, AT Thermistor".

14. Vaisala,"Users's Guide Vaisala Humidity and Temperature Probes HMP60 and HMP110 Series". 